\title{
Evidence for an S-velocity discontinuity in the lowermost mantle beneath the South Eastern Pacific Basin
}

\author{
Olivieri M., N.A. Pino and A. Morelli \\ Istituto Nazionale di Geofisica, via di Vigna Murata 605, 00143 Roma, Italy
}

\begin{abstract}
We used tangential component SH waveforms with $\mathrm{S}, \mathrm{ScS}$, sS and $\mathrm{sScS}$ arrivals of Chilean earthquakes recorded by four seismographic stations located in Antarctica to study the radial velocity structure at the core-mantle boundary (CMB) beneath the South Eastern Pacific basin. The selected station-event pairs give information on two distinct, close, patches of $D^{\prime \prime}$ beneath the Bellingshausen Sea. In one of these regions an anomalous arrival - interpreted as a reflection at the top of $D^{\prime \prime}$ (SdS phase) - is seen between $S$ and $\mathrm{ScS}$, together with early arrivals of ScS. This observation is well fit by adding to global reference model SP6 a reflector $315 \mathrm{~km}$ above the CMB, marking a $3 \%$ S-velocity increase. The new velocity model, usb2, fits both SdS and ScS travel times and amplitudes. Available data do not provide clear evidence to confirm or refute the presence of a similar velocity discontinuity in the second patch.
\end{abstract}

\section{Introduction}

The core-mantle boundary (CMB) plays a crucial role in many dynamical processes of the interior of the Earth, and it is target of vigorous research activity. In the past 15 years, seismology revealed important properties of the structure of the lowermost mantle. A notable feature is a $2-3 \% \mathrm{P}$ - and S-velocity discontinuity about $300 \mathrm{~km}$ above the CMB, which has been identified in several geographical regions: Loper and Lay [1995] summarize results on this topic from a variety of studies which have been conducted using different seismological techniques and data - such as PcP and ScS, their precursors, and $\mathrm{P}_{\text {diff }}$ or $\mathrm{S}_{\text {diff }}$. The $D^{\prime \prime}$ anomalous discontinuity does not appear to be a global feature, but rather a local one, as it has been identified only in selected regions and not found in others [Weber and Davis, 1990, Loper and Lay, 1995, Schlittenhardt et al., 1985]. Global tomographic studies do not have the resolution needed for detection of this sharp velocity increase. Broadband and short period body waveforms are well suited for these studies, as they permit investigation of structures from 10 to $500 \mathrm{~km}$ in scale [Loper and Lay, 1995]. As a consequence, our current knowledge is patchy. Areas of the CMB that have been already investigated mainly concentrate in the Northern hemisphere, where a better distribution of seismographic stations exists. This work investigates a new region of $D^{\prime \prime}$ in the southern hemisphere by observation of ScS precursors. Higher velocity just above the CMB causes shorter ScS-S differential travel times than global reference models. A sharp velocity discontinuity

Copyright 1997 by the American Geophysical Union.

Paper number 97GL52742.

0094-8534/97/97GL-52742\$05.00 at the top of $D^{\prime \prime}$ produces an arrival between S and ScS usually labelled SdS [Weber, 1993]. We use forward modelling to fit observed waveforms, for both direct (S, SdS, ScS) and depth phases (sS, sSdS, sScS). Since resulting evidence agree for these two groups of arrivals, in the following we concentrate on the direct $S$ wave train, but all considerations also hold for the sS train, even though, as pointed out by Lay [1986], a lower quality fit is to be expected for the surface reflected phases, given the lower signal-to-noise ratio and superimposed coda affecting these arrivals.

\section{Data}

The dataset is based on 8 Chilean earthquakes recorded by at least one of 4 close Antarctic seismographic stations located in the Ross Sea and Adelie Land areas. Their ray paths bottom beneath the Bellingshausen Sea in the South East Pacific basin (Table 1 and Figure 1). Many authors (e.g. Weber and Davis, 1990) suggest using only earthquakes deeper than $70 \mathrm{~km}$ to avoid superposition of direct and depth phases. However, it is also possible to reliably detect lower mantle anomalies from crustal events, even though this is less straightforward. Scarcity of deep earthquakes, and limited operational schedules of Antarctic seismographs installed in such a challenging environment only few years ago, limit the recording coverage for each event to at most 2 simultaneous stations. Epicentral coverage is between $66^{\circ}$ and $82^{\circ}$. Events with magnitude $m_{b}<5.5$, as well as those with apparent source complexity, were rejected because they do not allow clear recognition of the main phases arriving immediately after S.

\section{Evidence for an anomalous $D^{\prime \prime}$ layer}

The effective Fresnel zone at the core-mantle boundary for an $S$ wave with dominant period of about 6 seconds observed at a distance of about $80^{\circ}$ is an ellipse with axes $3.5^{\circ} \times 7^{\circ}[\mathrm{We}$ ber and Davis, 1990; Weber, 1993]. This is the area that contributes constructively to the formation of the reflected wave,

Table 1. CMT source parameters of events used in this work

\begin{tabular}{|c|c|c|c|c|c|c|c|}
\hline $\mathbf{N}$ & date & lat & lon & depth & time & $m_{b}$ & stations \\
\hline $\begin{array}{c}1 \\
2 \\
3 \\
4 \\
5 \\
6 \\
7 \\
8 \\
9 \\
10 \\
11\end{array}$ & $\begin{array}{lll}04 & 21 & 90 \\
05 & 14 & 90 \\
11 & 0492 \\
06 & 0893 \\
10 & 3093 \\
08 & 19 & 94 \\
09 & 12 & 94 \\
10 & 11 & 94 \\
02 & 14 & 95 \\
07 & 30 & 95 \\
11 & 01 & 95\end{array}$ & $\begin{array}{l}-36.95 \\
-35.84 \\
-31.63 \\
-31.59 \\
-31.75 \\
-26.65 \\
-31.13 \\
-32.11 \\
-23.29 \\
-23.32 \\
-28.90\end{array}$ & $\begin{array}{l}-73.25 \\
-71.38 \\
-71.55 \\
-69.22 \\
-68.21 \\
-63.38 \\
-71.64 \\
-71.44 \\
-67.70 \\
-70.56 \\
-71.20\end{array}$ & \begin{tabular}{|c|}
30.0 \\
81.0 \\
37.0 \\
113.0 \\
109.0 \\
565.0 \\
53.0 \\
51.0 \\
156.0 \\
33.0 \\
20.0
\end{tabular} & $\begin{array}{l}18: 54: 55.0 \\
21: 34: 04.8 \\
21: 32: 36.4 \\
23: 17: 41.4 \\
17: 59: 02.8 \\
10: 02: 51.8 \\
06: 29: 56.3 \\
01: 37: 20.8 \\
15: 53: 56.9 \\
21: 05: 50.6 \\
00: 35: 33.0\end{array}$ & $\begin{array}{l}6.0 \\
5.7 \\
5.8 \\
6.4 \\
5.9 \\
6.4 \\
5.8 \\
5.6 \\
5.7 \\
5.6 \\
6.3\end{array}$ & $\begin{array}{c}\text { DRV } \\
\text { DRV } \\
\text { DRV } \\
\text { DRV } \\
\text { DRV } \\
\text { SBA } \\
\text { DRV, SBA } \\
\text { DRV } \\
\text { TNV } \\
\text { SBA, VNDA } \\
\text { SBA }\end{array}$ \\
\hline
\end{tabular}




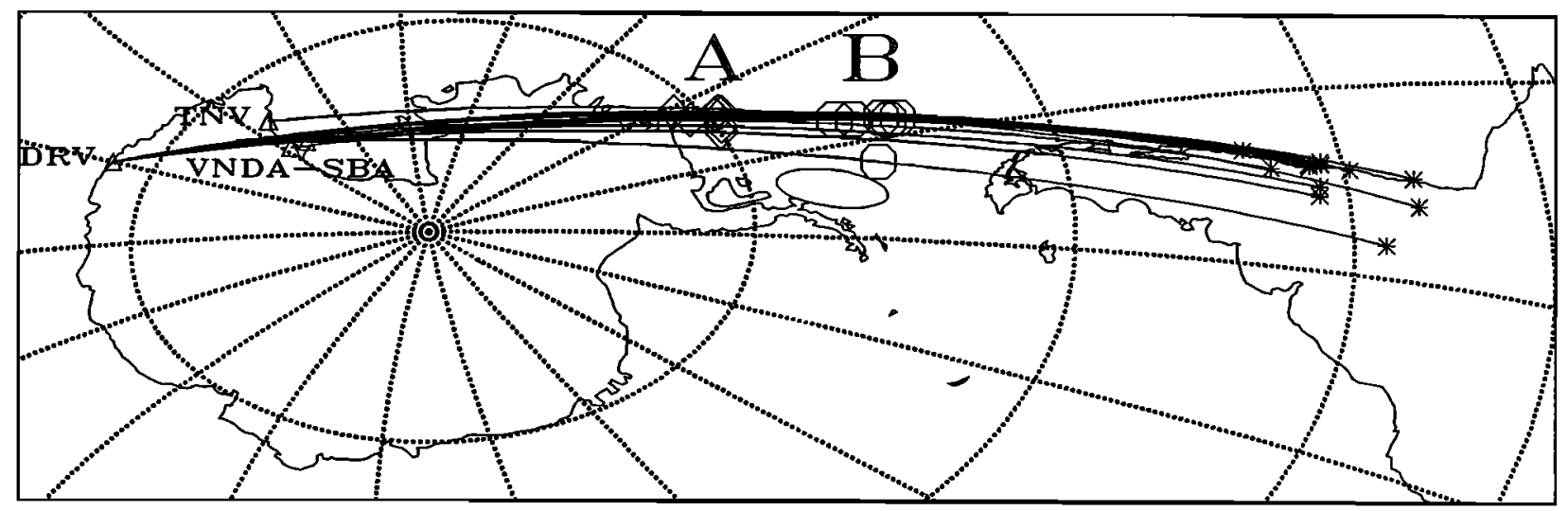

Figure 1. Geographical distribution of events $(*)$ and stations $(\Delta)$ used. Great circle paths are also plotted. The diamonds $(\diamond)$ represent reflection points at the CMB and the ellipse is the effective Fresnel zone ( $770 \mathrm{~km} \times 385 \mathrm{~km}$ wide). The projection is Mollweide.

and represents the resolution achievable by SdS waves. Figure 1 shows that our dataset can give information essentially on two, close, but separate patches at the CMB, identified as $A$ and $B$, which we must study separately. An important difference between the two datasets lies in the different range of epicentral distance. The majority of events bottoming in region $\mathrm{A}$ have epicentral distance between $78^{\circ}$ and $80^{\circ}$. In this range, SdS amplitudes increase with increasing distance, as the incidence angle becomes wider. SdS is most recognizable close to $80^{\circ}$, just before the $\mathrm{S}$ and ScS overlap. The dataset for region $\mathrm{B}$ consists of events with epicentral distance between $66^{\circ}$ and $77^{\circ}$. The seismograms have been integrated to yield displacement-proportional signal, and high-pass filtered at $0.01 \mathrm{~Hz}(0.02 \mathrm{~Hz}$ for event 4 in Table 1$)$. We only analyze the transverse component to avoid P-SV contamination.

\section{Reference model and role of crustal reflections}

Our reference model is SP6 [Morelli and Dziewonski, 1993]. We use centroid moment tensors [Dziewonski and Woodhouse, 1981] but adjusted focal depth to fit sS-S differential travel times. Synthetic seismograms computed with the Gaussian beam method [Červený and Pšenčík, 1983; Davis and Henson, 1993] show a good general agreement for absolute $\mathrm{S}$ travel times and phase amplitude ratios. Other global models, such as IASP91 [Kennett, 1991] and PREM [Dziewonski and Anderson, 1981], do not show significant differences in this range of distances.

Schlittenhardt et al. [1985] pointed out that the energy arriving between $\mathrm{S}$ and $\mathrm{ScS}$ onsets may be due to crustal effects such as top-side Moho reflections at the source (SmSS), or at the receiver (SSmS). Even though phase SSmS is always present, only crustal sources can originate SmSS. For a spherically symmetric Earth model, these two phases have the same travel time and polarity, and constructively interfere. In the distance range $65^{\circ}-85^{\circ}$ this results in a phase with amplitude similar to $\mathrm{ScS}$, travel time between $\mathrm{S}$ and ScS, but opposite polarity, which complicates recognition of phases originating in the vicinity of the CMB. As we use single stations, we must model crustal reflections - charac- terized by a different slowness than SdS. We achieved the best fit to SSmS and SmSS travel times by assuming a shallower $(27 \mathrm{~km})$ Moho than SP6 in the receiver region. Therefore, from now on our reference model will be a $2 D$ adaptation of SP6 with a thinner crust under the receiver (SP6'). However, we must point out that modelling the crust is not the main target of this work, and with these data alone the problem is under-constrained: crustal thickness and mean crustal velocity trade off. Therefore, the choice of thickness and velocity profile of the crust is only meant to account for the basic effects of the crustal structure. The crustal structure does not strongly influence the ScS-S differential travel time, which is shorter relative to SP6 in all of the seismograms, as shown in Figure 2 and Figure 3. Direct and depth phases (sS), for instance in event 5 of Figure 2, are well separated and both $\mathrm{ScS}$ and sScS arrive too early. This means that velocity in the lowermost mantle has to be higher than in SP6. Also a travel time analysis of our deeper events shows that both SSmS and ScS arrive too early after S when compared to synthetic seismograms. This observation cannot be fit by a crustal anomaly, as a thinner crust would accelerate SSmS, whereas ScS-S can only be modified by changing the model where the two ray paths differ, in the lowermost mantle.

\section{Evidence for an anomalous $D^{\prime \prime}$ layer}

Figures 2 and 3 show all the observations for regions $A$ and B, along with synthetics computed for model SP6, and for a new model, usb2, which is the result of forward modelling that we will presently discuss. ScS is always earlier than in SP6, requiring a change in the velocity profile in the lowermost mantle. Also, anomalous energy between $\mathrm{S}$ and $\mathrm{ScS}$ is present in data for region A. Some of these seismograms have background noise showing amplitude similar to this phase, but its frequency is apparently different and its occurrence is quite stable on seismograms recorded at similar distances (3-DRV, 8-DRV, and 4-DRV, 5-DRV, 7-DRV). This phase is unlikely to be a crustal reverberation since the shoulder arrival which is seen in the range $78^{\circ}-80^{\circ}$ degree is clearly not at the same time for the data at $73^{\circ}-75^{\circ}$. Moreover, even though the different overall shape of data and synthetics for event 3-DRV is likely to be an effect of source duration 
(the S pulse has evidently longer duration with respect to other data), the comparison of the waveform recorded for event 7 at station DRV (Figure 2) with the much simpler one recorded at SBA (Figure 3) clarify that the anomalous arrival cannot be associated to unmodeled source complexity as well. As suggested by Lay and Helmberger [1983] and Lay [1986], this feature can be reproduced by adding a sharp velocity increase in the lowermost mantle to produce the phase commonly known as SdS. The depth of the reflector and its velocity discontinuity can be constrained by the travel time and the amplitude ratio of SdS/S. Figure 4 shows model usb2, the result of forward modelling of seismograms of group $A$. usb2 differs from SP6 ${ }^{\prime}$ by the addition of a discontinuity at $2576 \mathrm{~km}$ depth ( $315 \mathrm{~km}$ above the CMB) with a $3 \%$ velocity contrast. Seismograms sampling the CMB in region $B$ have a lower signal-to-noise ratio, and even though they show ScS energy arriving earlier than in SP6', they do not show clear $D^{\prime \prime}$ reflections, neither SdS nor sSdS. This may be due to the smaller relative amplitude of these phases in this distance range. In some instances, superposition of different phases results in very complex seismograms where the main arrivals are not even clearly recognizable. In this region we can only infer the presence of a faster layer at the bottom of the mantle that causes the observed earlier $\mathrm{ScS}$ arrival times, but no clear conclusion can be drawn on the presence of a sharp discontinuity.

The slowness of a seismic phase can be ascertained by considering a profile of records as a function of distance from a given event. This technique requires the same event to be recorded by stations with different epicentral distances. Alternatively, different events recorded by the same seismographic station may be used, provided they all share the same focal depth. However, this condition may be approximated by slightly shifting the epicentral distance. For each event we use an equivalent epicentral distance, such as to make the observed ScS-S time coincide with the theoretical one, calcu-

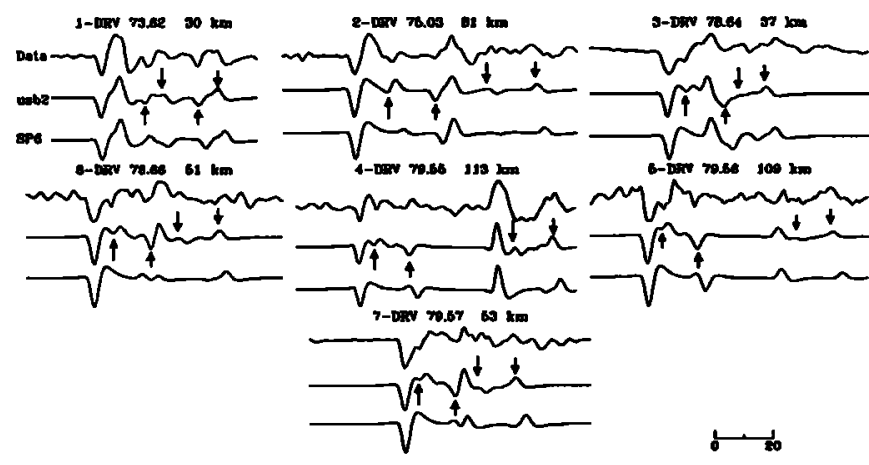

Figure 2. Transverse component (proportional to displacement) of events relative to region $\mathbf{A}$. The traces shown are (from top) the record displacement, two synthetic seismograms computed by using usb2 and SP6 as velocity model. Maximum peaks of arrivals are marked by arrows on the seismograms computed for our model. Upward arrows indicate direct phases (SdS, ScS), downward arrows mark depth phases (sSdS, sScS). Solid arrow tips point to $D^{\prime \prime}$ reflections, open tips denote CMB reflections. The events are identified by the number listed in table 1, the epicentral distance, in degrees, and the source depth, and recording station.
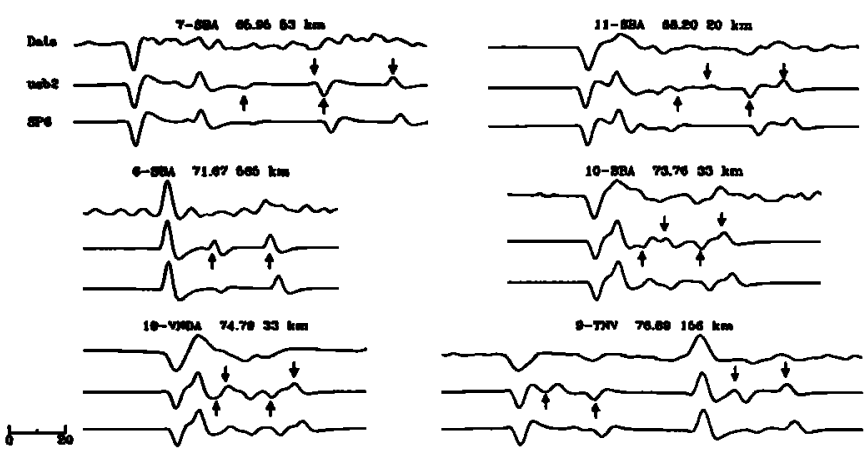

Figure 3. Events relative to region $B$, see figure 2 for symbols explanation.

lated for a reference source depth $(100 \mathrm{~km})$ and the proposed model. The result for seismograms traversing region $A$ is plotted in Figure 5. It can be noted that all inferred SdS arrivals are very close to their theoretical travel time branch. Their slowness confirms that this is the product of a deep reflection. We are also led to discard the possibility that the anomalous arrival is due to SKS energy (or SKKS) leaking to SH motion because of mantle anisotropy. Besides arriving closer to ScS than SdS does, SKS in fact has an appreciably smaller slowness than ScS and SdS.

Recent availability of seismograms recorded by stations installed in Antarctica allows use of shear wave ScS precursors to investigate the radial velocity structure in a region beneath the South East Pacific basin. Seismograms from earthquakes beneath the Chilean Cordillera, recorded by Ross Sea and Adelie Land stations, show SdS and sSdS arrivals originating along paths bottoming in our region $A$. Our preferred interpretation - that best models the anomalous $\mathrm{ScS}$ travel time, the ScS/S amplitude ratio and the SdS phase - involves the presence of a reflector located about $315 \mathrm{~km}$ above the core-mantle boundary, marking a $3 \% \mathrm{~S}$ velocity increase. Since SP6 and PREM at these depths display major differences only in $D^{\prime \prime}$, a very similar model would have resulted if we adopted the latter as reference, as revealed by synthetic

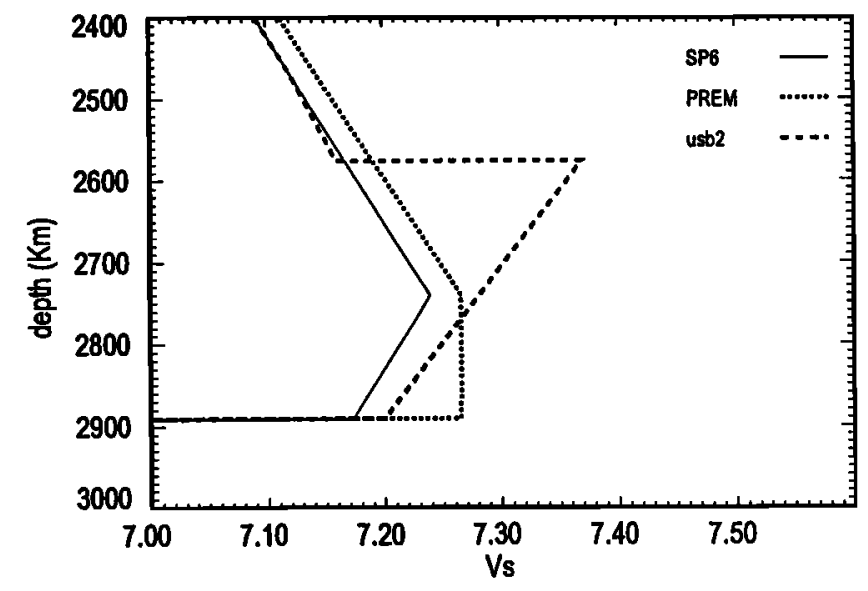

Figure 4. S-velocity profile of model usb2 in the lowermost mantle, compared to SP6 and PREM. 


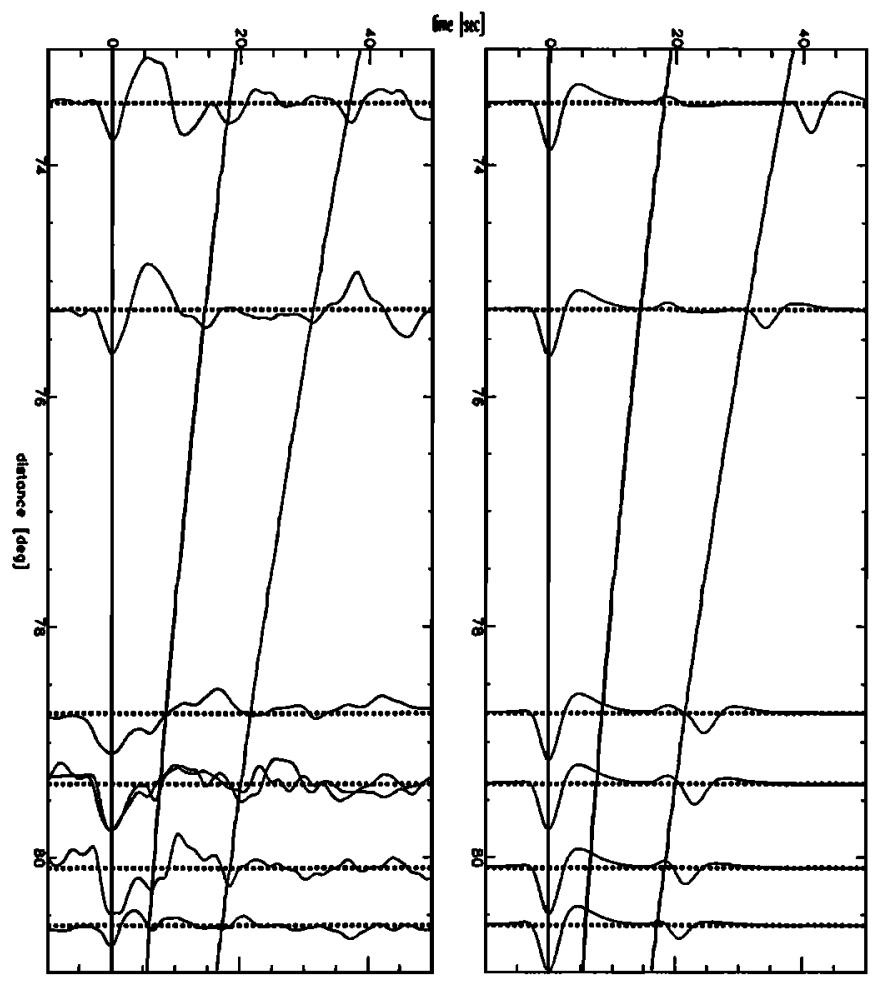

Figure 5. Profile section of the transverse component for data relative to region $A$, in the range of distance $72^{\circ}-82^{\circ}$. Left: data plotted at the equivalent epicentral distance (see text); right: synthetic seismograms computed for a source depth of $100 \mathrm{~km}$, by using SP6 and including S, ScS and SSmS. Reduced travel time curves are relative to $S, S d S$ and ScS for model usb2.

tests. Consistency of results obtained in modelling $8 \mathrm{ScS}$ and, for a couple of events, sSdS, is a confirmation of our interpretation. Instead, no evidence could confirm or disprove this hypothesis for rays bottoming in CMB region B, although this region appears to also have faster $S$ velocity at the bottom of the mantle. Recently, Kuo and Wu [1997] presented a global model of $D^{\prime \prime}$ lateral heterogeneity, obtained by inverting $\mathrm{S}_{\text {diff } f}-\mathrm{SKS}$ residuals. They show evidence for a 0.1 $\mathrm{Km} / \mathrm{s}$ faster $D^{\prime \prime}$ with respect to PREM in this region. This is sensibly higher than in our model. It should be noticed that their results are averaged on a lateral extension of $40^{\circ}$ at least in a $150 \mathrm{Km}$ thick $D^{\prime \prime}$. However, with our approach the velocity profile below the $D^{\prime \prime}$ reflector cannot be uniquely determined, as data only give information about the average velocity in the layer (the profile we impose is the simplest inference we can make), but the velocity at the bottom is constrained by the amplitude of ScS phase. We did not include heterogeneity in the lower mantle, such as Kuo and $\mathrm{Wu}$ [1997] did. However, an $S$ travel time anomaly of $2 \mathrm{~s}$ - as consequent to the model by Su et al. [1994] — would only yield a $40 \mathrm{~km}$ deeper discontinuity, a difference which could be considered as the error for our depth determination; while upper mantle heterogeneity would equally influence $S$, SdS, and $\mathrm{ScS}$ not affecting our results. From our analysis no inference is possible about the lateral extension and the shape of this lower mantle anomaly. More detailed studies will become possible as more data recorded in Antarctica will be available in the near future.

Acknowledgments. Work supported by PNRA (Programma Nazionale di Ricerche in Antartide). We thank $M$. Weber for discussions, T. Lay, B.Y. Kuo and J. Schweitzer for thorough reviews.

\section{References}

Cervený, V., and I. Pšenčík, Gaussian beams in two-dimensional elastic inhomogeneous media, Geophys. J. R. Astron. Soc., 72, 417-433, 1983.

Davis, J.P., and H. Henson, Development of an X-Windows tool to compute Gaussian beam synthetic seismograms, TGAL-9303,16 pp., Philips Lab., Hanscom AFB, MA, 1993.

Dziewonski, A., and D.L. Anderson, Preliminary reference Earth model (PREM), Phys. Earth Planet. Inter., 25, 297-365, 1981.

Dziewonski, A., and J.H. Woodhouse, An experiment in systematic study of global seismicity: Centroid Moment Tensor solutions for 201 moderate and large earthquake of 1981, J. Geophys. Res., 88, 3247-3271, 1981.

Kennett, B.L.N., IASPEI 1991 Seismological Tables, 167 pp., Australian National University, Canberra, 1991.

Kuo, B.Y., and K. Y. Wu, Global shear velocity heterogeneity in the $D^{\prime \prime}$ layer: inversion from Sd-SKS differential travel times, J. Geophys. Res., 1997, in press.

Lay, T., Evidence of a lower mantle shear velocity discontinuity in S and sS phases, Geophys. Res. Lett., 13, 1493-1496, 1986.

Lay, T., and D.V. Helmberger, A lower mantle S-wave triplication and the shear velocity structure of $D^{\prime \prime}$, Geophys. J. R. Astron. Soc., 75, 799-837, 1983.

Loper, D.E., and T. Lay, The core-mantle boundary region, $J$. Geophys. Res., 100, 6397-6420, 1995.

Morelli, A., and A. Dziewonski, Body wave travel times and a spherically symmetric P-and S-wave velocity model, Geophys. J. Int.112, 178-194, 1993.

Schlittenhardt, J., J. Schweitzer, and G. Müller, Evidence against a discontinuity at the top of $D^{\prime \prime}$, Geophys. J. R. Astron. Soc., 81, 295-306, 1985.

Su, W., R.L. Woodward, and A. Dziewonski, Degree 12 model of shear velocity heterogeneity in the mantle, J. Geophys. Res., 99, 6945-6980, 1994.

Weber, M., P- and S-wave reflections from anomalies in the lowermost mantle, Geophys. J. Int.115, 183-210, 1993.

Weber, M., and J.P. Davis, Evidence of a laterally variable lower mantle structure from P- and S- waves, Geophys. J. Int.102, 231-255, 1990.

Olivieri M., N.A. Pino and A. Morelli, Istituto Nazionale di Geofisica, Via di Vigna Murata, 605, 00143, Roma, Italy (e-mail: marco@atalante.ingrm.it)

(Received March 4, 1997; revised August 15, 1997; accepted September 18, 1997.) 